\title{
Discovering new host-directed therapies to treat
}

AUTHOR:

Stephanie Fanucchi ${ }^{1,2}$

\section{AFFILIATIONS:}

'Division of Chemical, Systems \& Synthetic Biology, Faculty of Health Sciences, University of Cape Town, Cape Town, South Africa

${ }^{2}$ Gene Expression and Biophysics Group, BTRI, Council for Scientific and Industrial Research - Biosciences, Pretoria, South Africa

\section{CORRESPONDENCE TO:}

Stephanie Fanucchi

\section{EMAIL:}

parrucchiere@mhlangalab.org

\section{KEYWORDS:}

immune response; sepsis; cancer; immunotherapy; IncRNA

\section{HOW TO CITE:}

Fanucchi S. Discovering new host-directed therapies to treat inflammation. S Afr J Sci. 2017;113(7/8), Art. \#a0224, 2 pages. http://dx.doi org/10.17159/sajs.2017/a0224

(c) 2017. The Author(s). Published under a Creative Commons Attribution Licence.
Dr Fanucchi is a South African born cell biologist who was recently awarded the 2017 L'Oreal/UNESCO International Rising Talent Award (15 of which are awarded worldwide annually); she was also a finalist for the 2016/2017 TW Kambule-NSTF Award for Emerging Researchers. Dr Fanucchi works as a senior scientist in Prof. Musa Mhlanga's laboratory; Prof. Mhlanga is the Director of the Biomedical and Translational Research Initiative, which is a new biomedical translational focused initiative lead by the Council for Scientific and Industrial Research and the University of Cape Town and funded by the Department of Science and Technology.

In 2003, an article published in Time magazine referred to inflammation as the 'silent killer'. It was around this time that medical doctors and scientists from different backgrounds started to realise that many disease states, including various cancers and autoimmune diseases, had an inflammatory basis. This important realisation revealed that we need to rethink how we treat these diseases. In particular, we need to carefully consider how inflammation impacts disease progression.

Inflammation is a so-called double-edged sword: you need it to clear infection and heal wounded tissues, but, if inflammation is not carefully regulated, it leads to many diseases such as various cancers, inflammatory bowel disease, rheumatoid arthritis and even sepsis. Sepsis or systemic inflammatory response syndrome is a serious inflammatory illness that is associated with a very high morbidity. There are 20-30 million sepsis cases reported each year, and it has been estimated that a sepsis-related death occurs every 3-4 s. ${ }^{1}$ The most common cause of sepsis is a bacterial infection, with viral and fungal infections contributing a smaller percentage. Once infected, the immune cells launch an attack to eliminate the infectious agent. The resultant inflammatory process helps the body to recover from the pathogen and repair damaged tissues. In a normal immune response, the body is able to precisely control the inflammatory response. However, in the case of sepsis and other inflammatory disorders, this process is not properly regulated, leading to the uncontrolled activation of the immune system and exacerbated levels of inflammatory processes. In these circumstances, the immune cells are not only targeting the infected or diseased tissue, but healthy tissue as well, leading to lethal consequences. Therefore, the ability to intervene and dampen excessive inflammation is the subject of intense research.

Cancer is another inflammatory-based disease that has a major influence on life expectancy. Every year, more than 14 million people worldwide are diagnosed with cancer. ${ }^{2}$ In South Africa, about 100000 people are diagnosed with cancer every year, with an average survival rate of $\sim 60 \%$ across all cancer types; with prostate, breast and colon cancer among the most prevalent. ${ }^{2}$ The immune system is composed of multiple cell types, which act synergistically to recognise and eliminate diseased or cancer cells. However, cancer cells are able to 'hide' from the immune system and in doing so are able to evade immune responses and escape eradication by immune cells. Recently, some exciting therapies have been able to 'unmask' immune cells so they are able to recognise cancer cells to kill them. These immunotherapies target immune checkpoint molecules and represent a promising new way to treat cancer. ${ }^{3}$ In some patients, these therapies have resulted in the successful reactivation of the immune system to kill the cancer cells, but unfortunately, this is not always the case. In other patients, immunotherapy leads to the overactivation of the immune system, elevated levels of inflammation and death. Clearly, current approaches to treat inflammatory disorders are not always successful. Thus there is a critical need to gain a detailed understanding of these processes, so we can develop new therapies and refine current ones.

At a cellular level, inflammation involves the sensing of the pathogen or disease causative agent which leads to the induction of signal transduction pathways. These pathways activate transcriptional regulators that switch on immune genes that encode various inflammatory mediators (e.g. cytokines). Therefore, inflammation is controlled at the level of gene regulation - which can be described in simple terms as the ability of genes to be switched on and off. This is a highly complex process that is not fully understood.

Major technical advances in biology are significantly advancing our understanding of gene regulation. For example, it took more than 10 years to sequence the human genome - a process that can now be completed in 1 to 2 days. Other important advancements include the ability to make discrete edits to DNA using gene editing tools, such as CRISPR/ Cas9 and the ability to use microscopy-based tools to visualise RNA and DNA at a single cell level. In the last decade, using a combination of these tools, scientists have made some very important discoveries in the field of gene regulation.

One surprising discovery is that the folding of DNA in the nucleus is not random. In almost every cell of the $\sim 1$ billion cells in the human body, there is a nucleus that contains DNA, or cellular blueprint. If you removed all the DNA from each nucleus and stretched it out, it would form a string longer than $1 \mathrm{~m}$. This DNA has to be packaged to fit inside the nucleus, which is one-fiftieth the size of a grain of sand. Therefore, a large quantity of DNA is packaged in a small space, and regions of DNA interact or 'kiss'. In a study in 2013, we showed that these 'gene kissing' interactions were important to the regulation of interacting genes. ${ }^{4}$ This study, combined with many others, revealed that we need to carefully consider how the folding of DNA in the nucleus impacts how genes are switched on. ${ }^{4,5}$ This is especially relevant in processes such as inflammation, which are ultimately controlled by how immune genes are switched on and off.

DNA can be transcribed, or made, into RNA which is then made into protein. Another surprising recent discovery was that the majority of genome is transcribed into RNA, and that not all of this RNA is made into protein. This subset of RNA is referred to as long non-coding RNA or simply IncRNAs. Thousands of IncRNAs are made, yet few have been characterised. Therefore, they represent an entirely new, exciting and unexplored area of drug targets. 
LncRNAs may act as intermediates that link information carried in the three-dimensional folding of DNA to gene regulation. Within the DNA sequence there are various types of regulatory or enhancer sequences that fine-tune gene expression. In certain circumstances, these regulatory sequences are located very far away from genes in onedimensional space. However, because of the compaction and looping of DNA, these enhancer elements can contact genes in three-dimensional space. Recently it has been shown that enhancer loci can be transcribed into a novel class of IncRNAs, termed enhancer RNAs (eRNAs). ${ }^{6}$ These eRNAs may regulate genes via diverse mechanisms that include the tethering of transcriptional regulators near to target gene(s). One of the greatest challenges associated with characterising IncRNAs is the inability to predict their function based on DNA sequence. Therefore, despite their abundance, a very low percentage of the thousands of annotated 'enhancer-derived' IncRNAs have been assigned a function.

Recently, we explored how IncRNAs and three-dimensional chromatin interactions regulate immune gene regulation. In this ongoing study, we identify a new class of 'enhancer-like' IncRNAs. We show that the three-dimensional folding of DNA in the nucleus brings these 'enhancerlike' IncRNAs in close proximity to immune genes, allowing them to 'kiss' and regulate the immune genes. Further, we have shown that it is possible to use small molecule inhibitors to 'drug' this response. As opposed to the commonly used strategy of targeting the pathogen, these drugs represent a new way to target host inflammation directly. Moving forward, we are collaborating with a number of pharma and biotech partners to test these inhibitors. Although these studies are still in very early stages, we are very hopeful that these drugs will be a new way to treat inflammation, and diseases such as cancer.

\section{References}

1. World Sepsis Day fact sheet [homepage on the Internet]. c2013 [cited 2017 Jun 28]. Available from: www.world-sepsis-day.org

2. Cancer Association of South Africa (CANSA). 2012 National cancer registry [homepage on the Internet]. c2012 [cited 2017 Jun 28]. Available from: http://www.cansa.org.za/south-african-cancer-statistics/

3. Sadelain M, Rivière I, Riddell S. Therapeutic $T$ cell engineering. Nature. 2017;545:423-431. https://doi.org/10.1038/nature22395

4. Fanucchi S, Shibayama Y, Burd S, Weinberg MS, Mhlanga MM Chromosomal contact permits transcription between coregulated genes. Cell. 2013;155:606-620. https://doi.org/10.1016/j.cell.2013.09.051

5. Dixon JR, Selvaraj S, Yue F, Kim A, Li Y, Shen Y, et al. Topological domains in mammalian genomes identified by analysis of chromatin interactions. Nature. 2012;485:376-380. https://doi.org/10.1038/nature11082

6. Andersson R, Gebhard C, Escalada IM, Hoof I, Bornholdt J, Boyd M, et al An atlas of active enhancers across human cell types and tissues. Nature. 2014;507:455-461. https://doi.org/10.1038/nature12787 